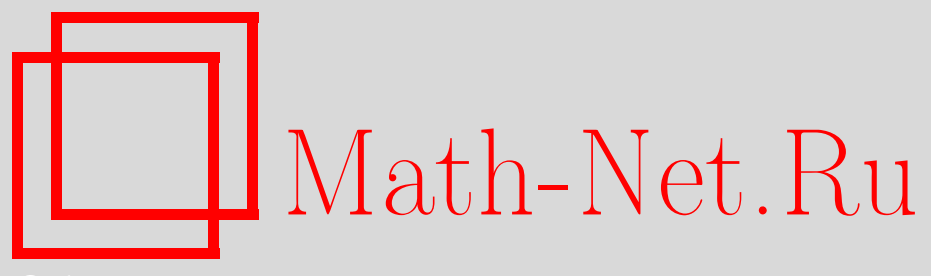

М. Ш. Шабозов, О наилучших квадратурных формулах для вычисления криволинейных интегралов на некоторых классах функций и кривых, Матем. заметки, 2014, том 96, выпуск 4, 637-640

DOI: https://doi.org/10.4213/mzm10239

Использование Общероссийского математического портала Math-Net.Ru подразумевает, что вы прочитали и согласны с пользовательским соглашением http://www . mathnet.ru/rus/agreement

Параметры загрузки:

IP : 3.80 .253 .173

26 апреля 2023 г., 14:51:11

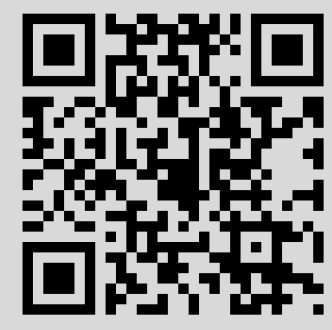




\section{О наилучших квадратурных формулах для вычисления криволинейных интегралов на некоторых классах функций и кривых}

\section{М.Ш. Шабозов}

В работе рассматривается задача о приближенном вычислении криволинейного интеграла первого рода для некоторых классов функций и классов пространственных кривых.

Пусть функция $f(M):=f\left(x_{1}, x_{2}, \ldots, x_{m}\right)$ определена и интегрируема вдоль кривой $\Gamma \subset \mathbb{R}^{m}$ и

$$
J(f ; \Gamma):=\int_{\Gamma} f(M) d t=\int_{\Gamma} f\left(x_{1}, x_{2}, \ldots, x_{m}\right) d t .
$$

Предположим, что на кривой Г установлено положительное направление, так что положение точки $M=M\left(x_{1}, x_{2}, \ldots, x_{m}\right)$ на кривой может быть определено длиной дуги $t=$ $\widetilde{A M}$, отсчитываемой от начальной точки $A$. Тогда кривая $\Gamma$ параметрически выразится уравнениями

$$
x_{1}=\varphi_{1}(t), \quad x_{2}=\varphi_{2}(t), \quad \ldots, \quad x_{m}=\varphi_{m}(t), \quad 0 \leqslant t \leqslant L,
$$

а функция $f(M)$ сведется к сложной функции $f\left(\varphi_{1}(t), \varphi_{2}(t), \ldots, \varphi_{m}(t)\right)$ от переменной $t$. В этом случае интеграл (1) запишется в виде

$$
J(f ; \Gamma)=\int_{0}^{L} f\left(\varphi_{1}(t), \varphi_{2}(t), \ldots, \varphi_{m}(t)\right) d t .
$$

Всякая квадратурная формула

$$
J(f ; \Gamma) \approx \mathcal{L}_{N}(f ; \Gamma ; P, T):=\sum_{k=1}^{N} p_{k} f\left(\varphi_{1}\left(t_{k}\right), \varphi_{2}\left(t_{k}\right), \ldots, \varphi_{m}\left(t_{k}\right)\right)
$$

для приближенного вычисления интеграла (3) задается векторами коэффициентов $P=$ $\left\{p_{k}\right\}_{k=1}^{N}$ и узлов $T=\left\{t_{k}: 0 \leqslant t_{1}<t_{2}<\cdots<t_{N} \leqslant L\right\}$, где $p_{1}, p_{2}, \ldots, p_{N}-$ произвольные действительные числа.

Погрешность квадратурной формулы (4) обозначим

$$
\left|R_{N}(f ; \Gamma ; P, T)\right|=\left|J(f ; \Gamma)-\mathcal{L}_{N}(f ; \Gamma ; P, T)\right| .
$$

Если $\mathfrak{M}$ - некоторый класс функций $\left\{f\left(\varphi_{1}(t), \varphi_{2}(t), \ldots, \varphi_{m}(t)\right)\right\}$, определенных в точках кривой Г и интегрируемых на отрезке $[0, L]$, то положим

$$
R_{N}(\mathfrak{M} ; \Gamma ; P, T)=\sup \left\{\left|R_{N}(f ; \Gamma ; P, T)\right|: f \in \mathfrak{M}\right\} .
$$

Пусть $\mathfrak{N}(L)$ - класс кривых Г, заданных уравнениями (2), длина которых равна $L$. Наибольшую погрешность квадратурной формулы (4) на классах функций $\mathfrak{M}$ и кривых $\mathfrak{N}(L)$ обозначим

$$
R_{N}(\mathfrak{M} ; \mathfrak{N}(L) ; P, T)=\sup \left\{R_{N}(\mathfrak{M} ; \Gamma ; P, T): \Gamma \subset \mathfrak{N}(L)\right\}
$$

Величину

$$
\mathcal{E}_{N}(\mathfrak{M}, \mathfrak{N}(L))=\inf \left\{R_{N}(\mathfrak{M} ; \mathfrak{N}(L), P, T):(P, T)\right\}
$$

будем называть оптимальной ощенкой погрешности формулы (4) на классах функций $\mathfrak{M}$ и кривых $\mathfrak{N}(L)$. Если существует вектор $\left(P^{0}, T^{0}\right)$, для которого

$$
\mathcal{E}_{N}(\mathfrak{M}, \mathfrak{N}(L))=R_{N}\left(\mathfrak{M} ; \mathfrak{N}(L), P^{0}, T^{0}\right),
$$

DOI: $10.4213 / \mathrm{mzm} 10239$ 
то этот вектор определяет наилучшую квадратурную формулу вида (4) в смысле Никольского [1] на классах функций $\mathfrak{M}$ и кривых $\mathfrak{N}(L)$.

Обозначим через $H^{\omega}:=H^{\omega}[0, L]$ множество функций $\varphi(t) \in C[0, L]$, удовлетворяющих условию

$$
\left|\varphi\left(t^{\prime}\right)-\varphi\left(t^{\prime \prime}\right)\right| \leqslant \omega\left(\left|t^{\prime}-t^{\prime \prime}\right|\right), \quad t^{\prime}, t^{\prime \prime} \in[0, L],
$$

где $\omega(\delta)$ - заданный модуль непрерывности, т.е. неубывающая полуаддитивная функция, в нуле равная нулю. Через $\bar{H}^{\omega_{1}, \ldots, \omega_{m}}[0, L]$ обозначим класс гладких кривых $\Gamma \subset \mathbb{R}^{m}$, заданных уравнениями (2), у которых координатные функции $\varphi_{i}(t) \in H^{\omega_{i}}[0, L], i=1, \ldots, m$. Если

$$
M^{\prime}=M\left(x_{1}^{\prime}, x_{2}^{\prime}, \ldots, x_{m}^{\prime}\right) \in \mathbb{R}^{m}, \quad M^{\prime \prime}=M\left(x_{1}^{\prime \prime}, x_{2}^{\prime \prime}, \ldots, x_{m}^{\prime \prime}\right) \in \mathbb{R}^{m},
$$

то введем в рассмотрение расстоянии

$$
\begin{aligned}
& \text { a) } \rho_{1}\left(M^{\prime}, M^{\prime \prime}\right)=\sum_{i=1}^{m}\left|x_{i}^{\prime}-x_{i}^{\prime \prime}\right| ; \\
& \text { b) } \rho_{2}\left(M^{\prime}, M^{\prime \prime}\right)=\left\{\sum_{i=1}^{m}\left(x_{i}^{\prime}-x_{i}^{\prime \prime}\right)^{2}\right\}^{1 / 2} ; \\
& \text { c) } \rho_{3}\left(M^{\prime}, M^{\prime \prime}\right)=\max _{1 \leqslant i \leqslant m}\left|x_{i}^{\prime}-x_{i}^{\prime \prime}\right| .
\end{aligned}
$$

Через $\mathfrak{M}_{\rho}$ обозначим класс функций $f(M)$, определенных на кривых $\Gamma \subset \bar{H}^{\omega_{1}, \ldots, \omega_{m}}$ и для любых двух точек $M^{\prime}, M^{\prime \prime} \in \Gamma$ удовлетворяющих условию

$$
\left|f\left(M^{\prime}\right)-f\left(M^{\prime \prime}\right)\right| \leqslant \rho\left(M^{\prime}, M^{\prime \prime}\right),
$$

где $\rho\left(M^{\prime}, M^{\prime \prime}\right)$ есть одно из перечисленных выше расстояний а)-с).

Таким образом, будем писать $f(M) \in \mathfrak{M}_{\rho_{1}}$, если для любых двух точек $M^{\prime}, M^{\prime \prime} \in \Gamma \subset$ $\bar{H}^{\omega_{1}, \ldots, \omega_{m}}$ и любых $t^{\prime}, t^{\prime \prime} \in[0, L]$ выполняется неравенство

$$
\left|f\left(M^{\prime}\right)-f\left(M^{\prime \prime}\right)\right| \leqslant \sum_{i=1}^{m}\left|x_{i}^{\prime}-x_{i}^{\prime \prime}\right|=\sum_{i=1}^{m}\left|\varphi_{i}\left(t^{\prime}\right)-\varphi_{i}\left(t^{\prime \prime}\right)\right| \leqslant \sum_{i=1}^{m} \omega_{i}\left(\left|t^{\prime}-t^{\prime \prime}\right|\right),
$$

а если $f(M) \in \mathfrak{M}_{\rho_{2}}$, то

$$
\left|f\left(M^{\prime}\right)-f\left(M^{\prime \prime}\right)\right| \leqslant\left\{\sum_{i=1}^{m} \omega_{i}^{2}\left(\left|t^{\prime}-t^{\prime \prime}\right|\right)\right\}^{1 / 2} .
$$

Сформулируем основной результат работы.

Теорема 1. Среди всех квадратурных формул вида (4) с произволвными векторами коэффициентов и узлов $(P, T)$,

$$
P=\left\{p_{k}\right\}_{k=1}^{N}, \quad T=\left\{t_{k}\right\}_{k=1}^{N}: \quad 0 \leqslant t_{1}<t_{2}<\cdots<t_{N} \leqslant L,
$$

наилучшей для классов функиий $\mathfrak{M}_{\rho_{i}}, i=1,2,3$, и кривых $\bar{H}^{\omega_{1}, \ldots, \omega_{m}}[0, L]$ является формула средних прямоугольников

$$
\int_{0}^{L} f\left(\varphi_{1}(t), \ldots, \varphi_{m}(t)\right) d t \approx \frac{L}{N} \sum_{k=1}^{N} f\left(\varphi_{1}\left(\frac{2 k-1}{2 N} L\right), \ldots, \varphi_{m}\left(\frac{2 k-1}{2 N} L\right)\right) .
$$

При этом для погрешности наилучшей формуль на классах функиий $\mathfrak{M}_{\rho_{i}}, i=1,2,3, u$ кривых $\bar{H}^{\omega_{1}, \ldots, \omega_{m}}$ справедливы точные оченки

$$
\mathcal{E}_{N}\left(\mathfrak{M}_{\rho_{1}} ; \bar{H}^{\omega_{1}, \ldots, \omega_{m}}\right)=(2 N) \sum_{i=1}^{m} \int_{0}^{L /(2 N)} \omega_{i}(t) d t
$$




$$
\begin{aligned}
\mathcal{E}_{N}\left(\mathfrak{M}_{\rho_{2}} ; \bar{H}, \omega_{1}, \ldots, \omega_{m}\right) & =(2 N) \int_{0}^{L /(2 N)}\left\{\sum_{i=1}^{m} \omega_{i}^{2}(t)\right\}^{1 / 2} d t \\
\mathcal{E}_{N}\left(\mathfrak{M}_{\rho_{3}} ; \bar{H}^{\omega_{1}, \ldots, \omega_{m}}\right) & =(2 N) \int_{0}^{L /(2 N)} \max _{1 \leqslant i \leqslant m} \omega_{i}(t) d t .
\end{aligned}
$$

ДокАЗАТЕльство. Не умаляя общности, докажем утверждение теоремы для классов функций $\mathfrak{M}_{\rho_{1}}$ и кривых $\bar{H}^{\omega_{1}, \ldots, \omega_{m}}$. Оценку снизу получим хорошо известным методом Корнейчука [2]. Через $\mathfrak{M}_{\rho_{1}, T}$ обозначим множество функций $f(M) \in \mathfrak{M}_{\rho_{1}}$, определенных вдоль кривой $\Gamma \subset \bar{H}^{\omega_{1}, \ldots, \omega_{m}}$, которые в узлах вектора $T=\left\{t_{k}\right\}_{k=1}^{N}$ обращаются в нуль

$$
f\left(\varphi_{1}\left(t_{k}\right), \ldots, \varphi_{m}\left(t_{k}\right)\right)=0, \quad k=1, \ldots, N .
$$

Фиксируем произвольный вектор узлов $T=\left\{t_{k}\right\}_{k=1}^{N}$ и определим кривую $\Gamma_{0}$ параметрическими уравнениями

$$
x_{i}=\varphi_{i}(t):=\min _{k} \omega_{i}\left(\left|t-t_{k}\right|\right), \quad i=1, \ldots, m, \quad 0 \leqslant t \leqslant L .
$$

Легко проверить, что функции $\varphi_{i}(t) \in H^{\omega_{i}}[0, L]$, а значит, $\Gamma_{0}$ принадлежит $\bar{H}^{\omega_{1}, \ldots, \omega_{m}}$. На кривой $\Gamma_{0}$ зададим функцию

$$
f_{T}\left(\varphi_{1}(t), \ldots, \varphi_{m}(t)\right)=\min _{t_{k}} \sum_{i=1}^{m} \omega_{i}\left(\left|t-t_{k}\right|\right)=\sum_{i=1}^{m} \omega_{i}\left(\min _{k}\left|t-t_{k}\right|\right),
$$

которая принадлежит классу $\mathfrak{M}_{\rho_{1}, T}$. В силу включения $\mathfrak{M}_{\rho_{1}, T} \subset \mathfrak{M}_{\rho_{1}}$ с учетом леммы 2 из [2] получаем оценку снизу погрешности формулы (4):

$$
\begin{aligned}
\mathcal{E}_{N}\left(\mathfrak{M}_{\rho_{1}} ; \bar{H}^{\omega_{1}, \ldots, \omega_{m}}\right) \geqslant \mathcal{E}_{N}\left(\mathfrak{M}_{\rho_{1}, T} ; \bar{H}^{\omega_{1}, \ldots, \omega_{m}}\right) \\
\quad=\inf _{(P, T)} \sup _{\Gamma \subset \bar{H}^{\omega_{1}, \ldots, \omega_{m}} \sup _{f \in \mathfrak{M}_{\rho_{1}, T}}\left|\int_{0}^{L} f\left(\varphi_{1}(t), \ldots, \varphi_{m}(t)\right) d t\right|} \quad \inf _{(P, T)} \int_{0}^{L} f_{T}\left(\varphi_{1}(t), \ldots, \varphi_{m}(t)\right) d t=\inf _{(P, T)} \sum_{i=1}^{m} \int_{0}^{L} \omega_{i}\left(\min _{k}\left|t-t_{k}\right|\right) d t \\
\quad \geqslant 2 N \sum_{i=1}^{m} \int_{0}^{L /(2 N)} \omega_{i}(t) d t .
\end{aligned}
$$

С целью получения оценки сверху, равной правой части (13), зададим квадратурную формулу (4) векторами коэффициентов и узлов соответственно

$$
P^{0}=\left\{p_{k}^{0}=L / N\right\}_{k=1}^{N}, \quad T_{0}=\left\{t_{k}^{0}=\frac{(2 k-1) L}{2 N}\right\} .
$$

Тогда для произвольной $f(M) \subset \mathfrak{M}_{\rho_{1}}$ и любой кривой $\Gamma \subset \bar{H}^{\omega_{1}, \ldots, \omega_{m}}$ будем иметь

$$
\begin{aligned}
& \left|R_{N}\left(f ; \Gamma ; P^{0}, T^{0}\right)\right| \\
& \quad \leqslant \sum_{k=1}^{N} \int_{(k-1) L / N}^{k L / N}\left|f\left(\varphi_{1}(t), \ldots, \varphi_{m}(t)\right)-f\left(\varphi_{1}\left(\frac{2 k-1}{2 N} L\right), \ldots, \varphi_{m}\left(\frac{2 k-1}{2 N} L\right)\right)\right| d t \\
& \quad \leqslant \sum_{i=1}^{m} \sum_{k=1}^{N} \int_{(k-1) L / N}^{k L / N} \omega_{i}\left(\left|t-\frac{2 k-1}{2 N} L\right|\right) d t=2 N \sum_{i=1}^{m} \int_{0}^{L /(2 N)} \omega_{i}(t) d t .
\end{aligned}
$$

Требуемое равенство (10) следует из сопоставления неравенств (13) и (14). По аналогичной схеме доказываются равенства (11) и (12). Теорема 1 доказана. 
В случае, когда $\omega_{i}(t) \equiv \omega(t), i=1, \ldots, m, 0 \leqslant t \leqslant L$, класс кривых $\bar{H}^{\omega_{1}, \ldots, \omega_{m}}([0, L])$ обозначим $\bar{H}_{m}^{\omega}[0, L]$. Из теоремы 1 как следствие получаем

Теорема 2. Среди всех квадратурных формул вида (4) с произвольными векторами коэффициентов и узлов $(P, T)$ наилучшей для классов функиий $\mathfrak{M}_{\rho_{i}}, i=1,2,3$, и кривых $\bar{H}_{m}^{\omega}[0, L]$ является формула средних прямоугольников. При этом для погрешности формулы на указанных классах функиий и кривых справедливы точные оченки

$$
\begin{aligned}
& \mathcal{E}_{N}\left(\mathfrak{M}_{\rho_{1}} ; \bar{H}_{m}^{\omega}[0, L]\right)=2 m N \int_{0}^{L /(2 N)} \omega(t) d t, \\
& \mathcal{E}_{N}\left(\mathfrak{M}_{\rho_{2}} ; \bar{H}_{m}^{\omega}[0, L]\right)=2 \sqrt{m} N \int_{0}^{L /(2 N)} \omega(t) d t, \\
& \mathcal{E}_{N}\left(\mathfrak{M}_{\rho_{3}} ; \bar{H}_{m}^{\omega}[0, L]\right)=2 N \int_{0}^{L /(2 N)} \omega(t) d t .
\end{aligned}
$$

\section{СПИСОК ЦИТИРОВАННОЙ ЛИТЕРАТУРЫ}

[1] С. М. Никольский, Квадратурнъе формуль, Наука, М., 1979. [2] Н. П. Корнейчук, Матем. заметки, 3:5 (1968), 565-576.

М.Ш. Шабозов

Поступило

Институт математики АН Республики Таджикистан

28.12 .2012

E-mail: shabozov@mail.ru

Исправленный вариант

17.02.2014 